\title{
Interactive comment on "32-year record-high surface melt in 2019/2020 on north George VI Ice Shelf, Antarctic Peninsula" by Alison F. Banwell et al.
}

\section{Anonymous Referee \#3}

Received and published: 26 November 2020

This is a well-written and evidenced paper that uses several independent datasets to explore the precise characteristics melting over the George VI Ice shelf. The authors demonstrate that melt extent and duration over GVIIS was higher in 2019/20 than in any other melt year analysed, and suggest that localised meteorological factors were responsible. Namely, persistent north-westerly and north-easterly low-speed winds, which allowed temperatures to rise above freezing and for melt to occur.

The manuscript is presented in a logical structure, with interesting and informative figures. The methods used are justified and largely given in sufficient detail to be reproducible. The results are supported by the data presented and the conclusions 
drawn are consistent with the evidence given. I recommend the publication of this manuscript in TC subject to minor revisions.

Specific comments:

[L49-52] You make reference to the collapse of Larsen A (and Larsen B in a previous sentence) and then in the next sentence to the partial collapse of Larsen A and B could you revise this to be clearer/more consistent with the above?

[L57-61] I think the 20.75 record has been thrown out by the WMO so you can probably remove this part of the sentence. The justification can be made using the Esperanza record alone.

[L62] You could link this back to the Bevan et al. paper again

[Section 3.4] Can you comment on the data processing for the AWS - e.g. is it an instantaneous value every 12 hours, or an average?

[L172] extra "to" after parenthesis

[para starting 187] Is there precedent for this type of methodology?

[para starting L320] It may be worth commenting on the fact that surface temperatures can differ from $2 \mathrm{~m}$ air temperatures and so 0 degrees at $2 \mathrm{~m}$ may not actually mean surface temperatures are at the melting point / vice versa. How might this influence the length of periods of $>0$ temperatures and consequently refreezing?

[para starting L335] During how much of the time series in Fig. 4a was the temperature above 1 standard deviation above the mean? This might be another interesting way to think about this anomalously warm period.

[para starting 335] Was any sensitivity testing conducted regarding the foehn detection method? Different thresholds and methods can yield very different results, e.g. isentrope-based method of King et al. (2017, doi: 10.1002/2017JD02680) vs surface method similar to the one used here. Varying the thresholds used can also make a 
difference (e.g. Turton et al., 2018, doi.org/10.1002/qj.3284).

Interactive comment on The Cryosphere Discuss., https://doi.org/10.5194/tc-2020-309, 2020.

Interactive

comment 